\title{
Mobile Data Exchange Model for Large-scale Campus Network based on EXI
}

\author{
Yu Chunyan \\ School of Computer and Information Engineering \\ Chuzhou University \\ ChuZhou, China \\ yuchy99@126.com
}

\author{
Wang Huibin ,Zhao Shenghui \\ School of Computer and Information Engineering \\ Chuzhou University \\ ChuZhou ,China \\ yuchy99@126.com
}

\begin{abstract}
In large-scale campus network, large amounts of mobile data exchange frequently. Transmission time and energy are consumed too much when smart mobile devices accessing the XML-based Web services. In this paper, a data exchange model is proposed which is based on EXI(Efficient XML Interchange) standard. This model is applied to smart mobile devices with limited resources. Finite state machine is used to describe the model formally and the process of EXI encoding is also analyzed. A campus mobile learning system was developed to check the efficiency of this model. Experiments shows remarkable reduction of transmission data, time and battery consume after EXI encoding.
\end{abstract}

Keywords: Mobile Data Exchange; Campus Network; Mobile Computing; EXI; XML

\section{INTRODUCTION}

In large-scale network, computing and services will bring about both large amount of interoperability and data exchange between heterogeneous systems, due to complex network structure and large number of users. Web service are currently widely used as service oriented architecture. Web services use XML (extensible Markup Language) in service description, data sharing and exchanging. When mobile intelligent devices access to Web Services or a large enterprise application Web Services, XML storage data, etc., efficiency will be significantly reduced due to the restriction of computing power, storage capacity, network access and the battery [1]. The high consumption of bandwidth and time will influence the users' experience, and also cause high communication costs. EXI(Efficient XML Interchange) is a new data exchange standard aimed at the limited resources environment and has been recommended by the W3C (World Wide Web Consortium) . EXI can be compatible with the current XML technology to the greatest extent and will reduce the consume of bandwidth, battery, processor and storage resources effectively at the same time ${ }^{[2]}$.

According to the characteristics of large-scale campus network, this paper introduces a campus mobile data exchange model suitable to the data exchange between intelligent devices and servers. This data exchange model is described in formalization description and EXI standard is adapted in this data exchange model to compress exchange data. Furthermore, mobile learning system was developed based on this model.

\section{RELATED WORKS}

Researchers have carried out many studies on data exchange based on $\mathrm{XML}^{[3,4,5]}$ involving focusing on the conversion between XML and relational database, using ontology to make XML data model with structure information and semantic information, applications in specific fields.

In recent years, with the wide application of XML in Web services, researches were carried out in the Web services of mobile data exchange. EXI was used in some of those researches ${ }^{[6,7]}$. For example, compression techniques ${ }^{[6]}$ of Web service in mobile network is studied. Researchers in university of Finland aalto ${ }^{[7]}$ analyzed different optimization methods such as HTTP compression, cache and agents in order to save energy consumption of mobile solutions.

Literatures $^{[8-11]}$ focus on using EXI to improve the transmission efficiency in mobile networks and the Internet of things. Sebastian ${ }^{[8]}$ studied the data exchange technology in sensor networks and designed the EXI processor to transform an XML document and EXI data flow,.

Joana $^{[9]}$ studied several compression methods such as Gzip, EXI to ensure the efficiency of Web service in instable military network. Angelo ${ }^{[10]}$ applied EXI to the binary Web service running on temperature and humidity sensor nodes to achieve high memory utilization. Li Jianxing ${ }^{[11]}$ studied the optimization approach of Web services using EXI to encode Web service SOAP message and servlets and tested in the simulation environment.

literatures ${ }^{[12,13]}$ were studied on the data exchange model based on XML. Shi Yanhua ${ }^{[12]}$ proposed a dynamic data exchange model between the government, enterprises. The model can support the change rules of data exchange following four steps to exchange data, that is service registration, agent, management and exchange. The model did not take into account the data exchange in mobile environment and ignored the analysis of data exchange efficiency. Chen Shengbo ${ }^{[13]}$ carried out study on modeling 
and verifying Web applications by FSM and analyzed the consistency and security of navigation. This research did not include the data exchanging on mobile devices.

As mentioned above, further research should be carried out on the exchanging data in practical mobile or embedded environment, such as the efficiency and the practical implement model. On the basis of research mentioned above, this paper analyzed the objects in large-scale campus network and put forward a model for mobile data exchanging in large-scale network which is described in formalization. Finally, A mobile learning system was developed based on CMED model and experiment was carried out to verify the performance of the model.

\section{MOBILE SERVICE IMPLEMENT MODEL}

In large-scale campus network, thousands of users will be connected to the internet in the same time, and one user may have several mobile terminals, which will cause a large number of data exchanging. However communication cost of $3 \mathrm{G}$ network is relatively high and many resources can only be available for the users in campus network. In addition, the bandwidth is limited for so many concurrent users and it is difficult to guarantee the stability of the high speed wireless transmission in a building with complex architecture, therefore the compressed data Web service is an effective way to improve network performance

\section{A. Mobile Data Exchange Architecture of Large-scale Campus Network}

Based on the campus network and application, under the network environment, mobile data exchange system is mainly composed of the communication circuit, network equipments, mobile intelligent devices, sensors, servers and storages, as shown in figure 1.The servers includes the database servers, Web servers, the UDDI registry servers, etc. and each server can be a physical server or a virtual machine. In the campus environment, clients often know the URL to invoke the Web service therefore the UDDI server only need to provide simple registration service and directory service.

Mobile data exchange process includes: (1) The Web service provider, in the form of a server, register in UDDI (Universal Description, Discovery and Integration) server through wired network.(2) Mobile intelligent device send a request to UDDI, and UDDI looks up for the right service and send back the WSDL document to the mobile client.(3)Mobile intelligent device generate SOAP message from the WSDL document and then send the SOAP request to the Web service provider.(4) The Web service provider executes operation according to the SOAP request and then generate the SOAP response document which will be sent back to the mobile device.

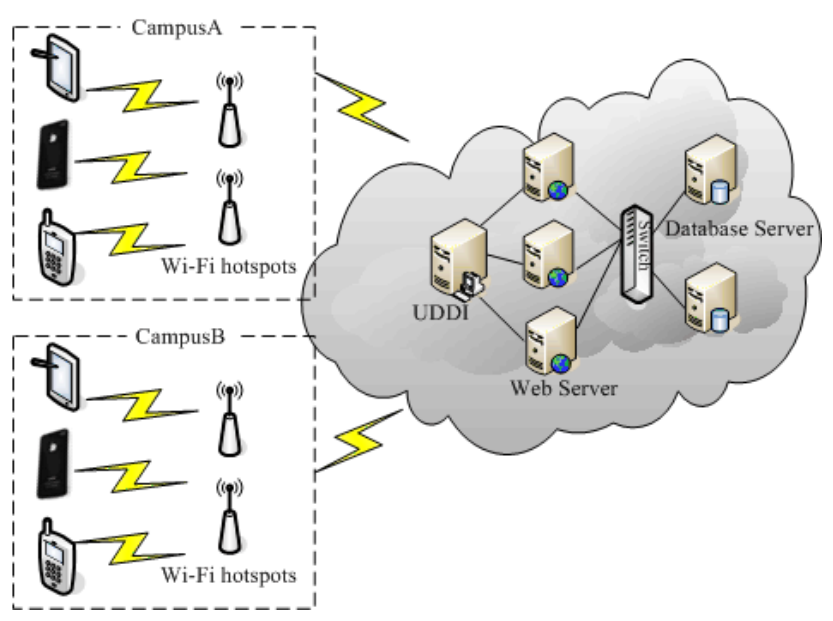

Fig. 1 mobile data exchange system in large-scale campus

\section{B. Campus Mobile Data Exchange(CMDE) Model based on EXI}

Mobile Data Exchange model (Campus Mobile Data Exchange) describes process of Data Exchange between the mobile intelligent device and the Web service provider. CMDE includes resource management layer, SOAP processing layer, EXI processing layer, HTTP processing layer and transport layer, as shown in figure 2. Different from the traditional Web services exchange model, EXI processor works both on the server side and the mobile client side, which covert the XML documents into the EXI stream before transmission on the internet and convert the EXI stream into XML documents before take operation based on the document content.

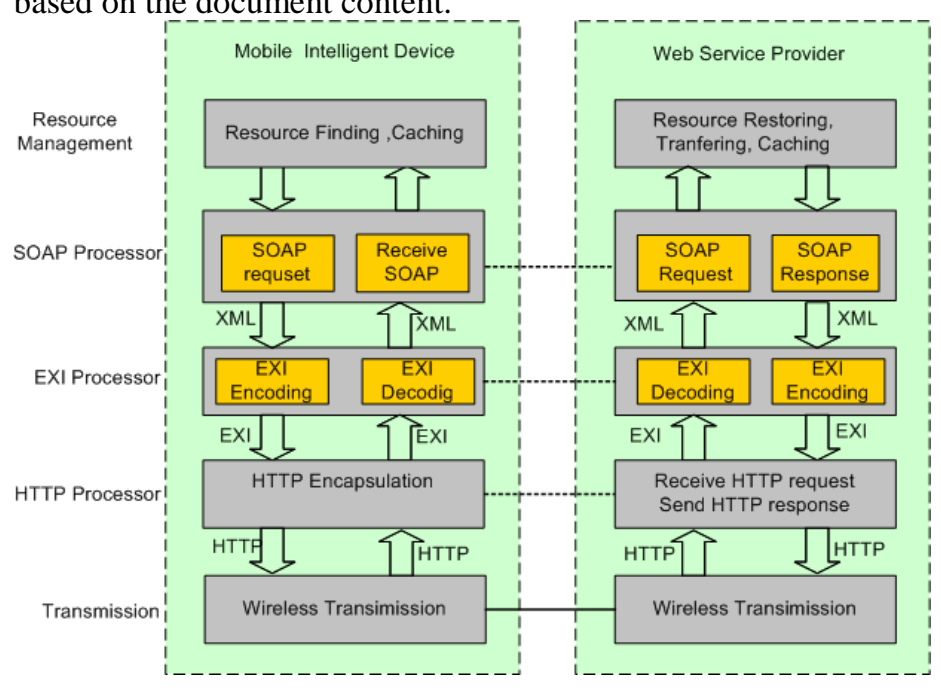

Fig.2 data exchange between the clients and servers

\section{Formalization Description of CMDE Model}

In order to describe accurately and realize this model, CMDE system can be expressed as an algebraic system composed of object, operation of object and constrains of 
operation. In order to analyse the system, some definition are given as follows.

Definition 1: mobile data exchange system, is an abstract algebraic system which includes the object, the operation of object and operating constraints. It is represented as $\mathrm{S}(\mathrm{Ob}$, $\mathrm{Op}$, Cons). $\mathrm{Ob}$ is the set of objects, Op is the set of operations taken by these objects, and Cons is set of the constraints of the operation.

Definition 2: object, stands for each component in the system as listed below:

MClient(mobile intelligent device browser),

HServer(HTTP server),

UServer(UDDI server),

WNetwork(wireless communication network),

SProcessor(SOAP processor),

EProcessor(EXIprocessor),

SRequest(SOAP request message),

SResponse(the SOAP response message),

HRequest(HTTP request message),

HResponse(HTTP response message),

EDoc(EXI document).

So object can be described as follows.

$\mathrm{Ob}=\{$ HServer,MClient,UServer,WNetwork,SProcessor,E

Processor,SRequest,SResponse,HRequest,HResponse,EDoc

Definition 3: operation of the object, is the set of operations and can be represented as follows:

HServer.Op: send registry request, receive HTTP message, caching, send HTTP response, receive SOAP request, send SOAP response.

Mclient.Op: send request to service lookup, accept the result of the service lookup, send SOAP request, receive SOAP response, send HTTP message, receive HTTP message.

WNetwork.Op: transfer HTTP message.

UServer.Op: service register, service lookup, send WSDL document.

SProcessor.Op: receive SOAP request, send the SOAP response.

EProcessor.Op: receive XML document, receive EXI document, encoding, decoding, sending XML document, receiving EXI document.

So, operation on the object can be expressed as follows. $\mathrm{Op}=\{$ HServer.Op,Mclient.Op,WNetwork.Op,UServer.Op, SProcessor.Op,EProcessor.Op\}.

$\mathrm{FSM}^{[13]}$ (Finite State Machine) is one of the important ways for formal representation, which can accurately describe the different sequences of the system and how to respond to external events. The data exchange process between the mobile client and the server in CMDE model could be described by FSM since that the system has definite numbers of states and the next state will be determined by the current state and the input information. Define CMDE model as a tuple:

$\mathrm{CMDE}=(\mathrm{S}, \mathrm{f}, \mathrm{S} 0, \mathrm{Z})$

$\mathrm{S}$ is the set of states, $\mathrm{F}$ is a transformation function, $\Sigma$ is the set is of Input information, $\mathrm{S} 0$ is the initial state, $\mathrm{Z}$ is the final state, $\mathrm{S} 0, \mathrm{Z} \in \mathrm{S}, \quad \sum=$ \{SRequest,SResponse,HRequest,HResponse,EDoc $\}, S=\{\mathrm{S} 0$, S1,S2,S3,S4,S5,S6,S7,S8,S9,S10\},

S0 (wait) : MClient gets resquest from the UServer, and ready to send the service request to the HServer.

S1 (SOAPRequest): MClient using SOAP message processing middle-ware generate SOAP message in the format of XML.

S2 (EXIEncode): EProcessor of MClient encodes XML document to EXI document.

S3 (HttpRequest): MClient packages the EXI document into HTTP message, and then sends the HTTP message to a Web server.

S4(Transmit): wireless network transmit the HTTP message.

S5 (HttpRecieve): HServer receives HTTP message, and parses the EXI document from the HTTP message.

S6 (EXIDecode): HServer decodes EXI document into XML document.

S7 (SOAPResponse): HServer does operations such as database processing according to the request information in the SOAP envelope, and wraps the SOAP response in the format of XML.

S8: (EXIEncode): Eprocessor of HServer encodes the XML documents into EXI documents.

S9(HttpResponse): HServer wraps the EXI document into HTTP message and then sends HTTP message to Mclient.

S10 (EXIEncode): Mclient parses the EXI document from the HTTP message.

S11 (EXIEncode): Mclient decodes EXI documents to XML document and display with the style file .

The state transition diagram of CMDE model is shown in figure 3 .

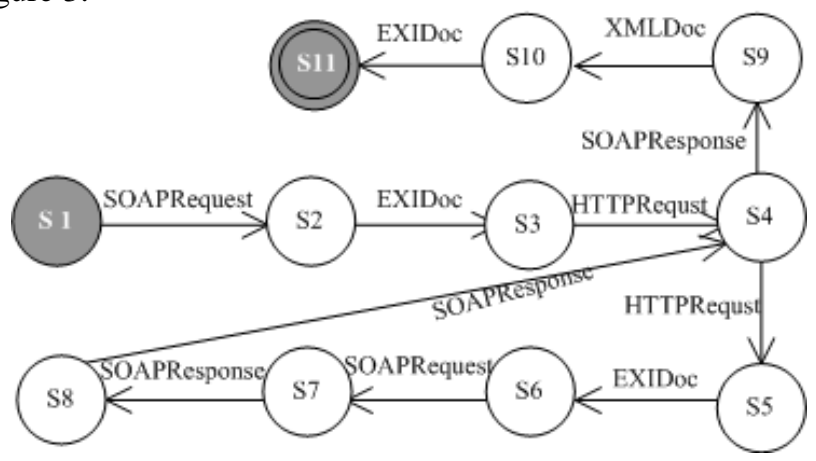

Fig.3 state transition diagram of CMDE model

\section{EXI Encoding Process}

EXI processing layer is the core of the CMDE model is, which work at mobile intelligent device and server. The EXI processor is composed of EXI encoder and decoder to compress XML document to the EXI document uncompress the EXI document to the XML document. We illustrate the EXI encoding process using the following SOAP response document for an example.

Code List 1 : SOAP response document

$<$ ?XML version="1.0" encoding="utf-8" ?> 
$<$ soap12:Envelope

xmlns:xsi="http://www.w3.org/2001/XMLSchema-instance" xmlns:xsd="http://www.w3.org/2001/XMLSchema"

xmlns:soap12="http://www.w3.org/2003/05/soap-

envelope">

<soap12:Body>

$<$ MLearningRes xmlns="http://learning.chzu.edu.cn/">

$<$ Course CourseNo="203006" >

$<$ TeacherName>Chen Min</TeacherName>

$<$ Lecture $><$ name $>$ Lecture 1 introduction $</$ name $>$ $<$ url $>203006$ ไhandout $\backslash 1 . p p t</$ url $></$ Lecture $>$

$<$ Lecture $><$ name $>$ Lecture 2 markup language $</$ name $>$ $<$ url $>203006$ handout 2 .ppt $<$ /url $></$ Lecture $>$

$</$ Course $>$

$</$ MLearningRes $>$

$</$ soap12:Body $>$

$</$ soap12:Envelope $>$

EXI processor could compress XML documents into EXI documents without loss quickly with the following steps ${ }^{[14]}$ :

(1)According to the EXI grammar, the XML document will be encoded into two parts, the head stream and body stream. XML documents can be encoded in built-in grammar pattern(without XML schema), or in Schema-informed grammar pattern(with XML schema). Optimization strategy is used in Schema-informed grammar model and bring higher processing efficiency.EXI document includes the EXI header and EXI Body. EXI Header can distinguish EXI streams from text XML documents, identify the version of the EXI format, and specify the options used to process the body of the EXI stream ${ }^{[1]}$.

The EXI cookies (optional, used to distinguish the EXI flow with other data flow), Distinguishing Bits (expressed as "10", to distinguish from the XML document), Presence of Bit for EXI Options, EXI Format Version (EXI Version of data flow), EXI Options (code and the various Options, such as whether to retain them, etc.), Padding Bits (optional, bytes compressed mode fill $\mathrm{N}$ bytes), etc.

EXI Body is the stream of the compressed XML document and consists of a series of event stream and the data content. XML document is composed of tags, attributes, attribute values, data contents, comments, PCDATA and so on. Tags, attributes are encoded as structural information, while attribute values and data contents are encoded as content information. The structural information will be encoded into specific event stream and the content information will be encoded into temporal table and then be encoded through replacing the repeated content with the sign of the content. For example, the start tag of XML document is notated as SD, the end tag of XML document is notated as $\mathrm{EE}$, the start tag of element is notated as SE, the end tag of element is notated as EE, the attribute is notated as AT. Take the SOAP response document as an example to illustrate the EXI event stream encoding process in figure 4.

(2) pre-compression. Separate the structure information and content information and then rearrange in the form of an aligned sequence for quick compression.
(3) compression. Compress the event steam into binary information, and compressed tag name and element value only appears once.

The algorithm shown blow is how to encode XML document into EXI event stream.

Do While() \{

1 Get the next event data to be encoded If (schema is available) \{

2 us schema-informed pattern

3 read schema

4

Else \{ go to 5

not processed) \{

If not(Fidelity option indicates this event type is

Use the grammars to determine the event code of the event Encode the event code followed by the event content Evaluate the grammar production matched by the event \})

\section{APPLICATION EXPERIMENT}

In order to check the model in practice, a "mobile learning management system based on the EXI" was developed. The prototype system provides the following Web services: login and authentication, user information modification, browsing the course list, browsing the resource list of a specific course, downloading the curriculum resources such as PPT, DOC, PDF documents, rating on course materials, etc.

Developing: We developed two EXI processors deployed on smart phones and the server utilizing the open source software OpenEXI ${ }^{[15]}$. EXI processor on smart phone was developed for android system and processor on the server was developed in Java for axis2.

Experiment environment: smart phone in campus A connected to the Web server of campus B. The server was configured to Intel Xeon2.2 G, 4G memory, JDK1.7, Tomcat6.0.36, axis2. The router was TPLink WR745n and HTC T328t was chosen as the mobile smart device of android 4.3, 512M,2G.

Purpose and methods: To compare the common Web services to Web services based on CMDE model by transmitting document size, time consumption, power consumption of mobile phone. According to the former analysis of CMDE model, CMDE model provides smaller size of transmit information which will shorten the transmission time, but needs some time to do encoding and decoding which will cause the consumption of time and power.

Experiment process: Smart phone was used to visit 5 Web services. TCPMonitor was used to see the size of the SOAP document, request and response time, and the BatteryLeft was used to monitor the Left power of the battery. Firstly, each Web service was tested for 50 times and each time we recorded the total time of each invoking Web service ( from sending request to receiving response), the time of each transmission and the left power of battery. 
Secondly, each compressed Web service was tested for 50 times and each time we recorded the size of EXI documents, the EXI encoding time and decoding time on mobile phone, transmission time, and checked the left power of battery after finishing test. Thirdly, we calculated the average value of those data above. The test results are shown in table 1, in which SizeX(the unit is bit) stands for the size of the SOAP document, SizeE stands for the size of the SOAP document after compression. TimeX(the unit is $\mathrm{ms}$ ) stands for $\mathrm{Web}$ service invoking time. TimeE stands for Web service invoking time based CMDE. TimeE-1,TimeE-2,TimeE-3 respectively stand for the encoding time, decoding time and the transmission time based on CMDE. PowerX stands for the left energy of the battery(the unit is mv) after invoking the Web service for 50 times, PowerE stands for energy consumption of invoking Web services based on EXI.

TABLE I. EXPERIMENT Results OF INVOKING Web SERVICE (1)

\begin{tabular}{cccccc}
\hline id & SizeX & SizeE & TimeX & TimeE & TimeE-1 \\
\hline 1 & 1012 & 702 & 795 & 664 & 341 \\
2 & 25993 & 22420 & 1973 & 2293 & 1379 \\
3 & 51817 & 4255 & 2918 & 2803 & 493 \\
4 & 266682 & 72971 & 9387 & 9734 & 3761 \\
5 & 395746 & 341067 & 15418 & 15211 & 7601 \\
\hline
\end{tabular}

TABLE II. EXPERIMENT RESULTS OF INVOKING WEB SERVICE (2)

\begin{tabular}{cccccc}
\hline id & SizeX & TimeE-2 & TimeE-3 & PowerX & PowerE \\
\hline 1 & 1012 & 240 & 83 & 4323 & 4312 \\
2 & 25993 & 749 & 165 & 4210 & 4217 \\
3 & 51817 & 341 & 1969 & 4276 & 4211 \\
4 & 266682 & 1673 & 4300 & 4233 & 4229 \\
5 & 395746 & 1108 & 6502 & 4215 & 4173 \\
\hline
\end{tabular}

Experiment data shows that size of transmission data reduced remarkably after encoding into EXI stream,and the transmission time also decreased efficiently. There was little different in battery consumpt between the general Web service model and the CMDE model. The time of encoding and decoding occupied a large proportion of the time of entire invoking process so that TimeX is little different from TimeE.

\section{CONCLUSION AND FUTURE WORK}

According to the characteristics of the large-scale campus network, a mobile data exchange model was put forward and was analyzed in formal description. A mobile learning system based on the mobile data exchange model was also developed in practice and contrast experiment had been taken to check the efficiency of the model. Next step is to improve the efficiency of the SOAP message with attachments, reduction of EXI processing time on the mobile devices and enhance the overall performance of the data exchange system.

\section{ACKNOWLEDGEMENT}

This research was financially supported by the Natural Science Foundation of Anhui Province Department of Education.

\section{REFERENCES}

[1] Information on: http://www.w3.org/TR/exi/.

[2] Information on: http://www.w3.org/TR/2004/WD-xbc-usecases-20041109/.

[3] Marcelo Arenas, Pablo Barcelo, Leonid Libkin. First Editon XML-Managing Data ExchangeM.Morgan \& Claypool Publishers ,2011.

[4] Hossam Jumaa, Paul Rubel, Jocelyne Payn.An XML-Based Framework for Automating Data Exchange in Healthcare[C].eHealth Networking Applications and Services (Healthcom), 2010 12th IEEE International Conference .

[5] Peng Tao,Zhang Li.Ontology and XML based data exchange system : submitted to Journal of computer engineering (2006).

[6] Lars Johnsrud, Dinko Hadzic, Trude Hafsoe. Efficient Web Services in Mobile Networks[C]. Sixth European Conference on Web Services,2008:197-204.

[7] Le Wang, Edward Mutafungwa, Yeswanth Puvvala, Jukka Manner. Strategies for Energy-Efficient Mobile Web Access: An East African Case Study. AFRICOMM 2011, LNICST 92, pp. 74-83, 2012.

[8] Sebastian Kabisch, Daniel Peintner, Jorg Heuer. Efficient and Flexible XML-based Data-Exchange in Microcontrollerbased Sensor Actor Networks.[C]. 2010 IEEE 24th International Conference on Advanced Information Networking and Applicating Workshops.508-513.

[9] Joanna Śliwa,Tomasz Podlasek,Marek Amanowicz. Web Services Efficiency in Disadvantaged Environment: submitted to Journal of. Telecommunications and Information Technology,2011.

[10] Angelo P. Castellani,Nicola Bui,Paolo Casari. etc. Architecture and Protocols for the Internet of Things-A Case Study[C]Proceedings of 2010 8th IEEE International Conference on Pervasive Computing and Communications Workshops, 2010.

[11] Li Jianxing.A research of EXI-based Web service optimization approach[D].Guangzhou:South China university of technology,2010.

[12] Shi Yanhua, Li shiyu.Research of data exchange model based on dynamic service :submitted to Journary of applicaton research of computers, 2011.

[13] Chen Shengbo. Modeling and verifying Web applications[D]. Shang Hai:ShangHai university doctoral dissertation, 2009 .

[14]Information on :http://www.w3.org/TR/2009/WD-exiprimer-20091208.

[15] Information on :http://openexi.sourceforge.net 